\title{
Shannon Sampling in the Clifford Analysis Setting
}

\author{
Kit-Ian Kou and Tao Qian
}

\begin{abstract}
The paper concerns the generalization of the one-dimensional sinc function to $(n+1)$-real variables within the Clifford analysis setting. The exact interpolation with Shannon sampling is proved.
\end{abstract}

Keywords: Shannon sampling, sinc function, Clifford analysis, monogenic functions, harmonic analysis

MSC 2000: Primary 62D05, secondary 30D10, 11E88, 30A05, 42B35

\section{Introduction}

The sinc function on the real line $\mathbb{R}$ is

$$
\operatorname{sinc}(x)=\frac{1}{2 \pi} \int_{-\pi}^{\pi} e^{\mathbf{i} x t} d t=\frac{\sin (\pi x)}{\pi x} .
$$

It has a holomorphic extension to the complex plane $\mathbb{C}$ :

$$
\operatorname{sinc}(z)=\frac{1}{2 \pi} \int_{-\pi}^{\pi} e^{\mathbf{i} z t} d t=\frac{\sin (\pi z)}{\pi z} .
$$

Systematic studies on sinc function in one complex variable and applications to numerical solutions for problems in partial differential equations and geometry may be found in the work of Stenger [11] and Lund et al. [5].

For a set $A$, let $\mathcal{X}_{A}$ denote the characteristic function of $A$. Sinc function in $\mathbb{R}^{n}$ is defined by

$$
\operatorname{sinc}(\underline{x})=\frac{1}{(2 \pi)^{n}} \int_{\mathbb{R}^{n}} e^{\mathbf{i}\langle\underline{x}, \underline{\xi}\rangle} \mathcal{X}_{[-\pi, \pi]^{n}}(\underline{\xi}) d \underline{\xi} .
$$

Kit-Ian Kou and Tao Qian: Department of Mathematics, Faculty of Science and Technonogy, University of Macau, Macau; kikou@umac.mo and fsttq@umac.mo The study was supported by Research Grant of the University of Macau No. RG021/03-04S/QT/FST

ISSN 0232-2064 / \$2.50 C Heldermann Verlag Berlin 
The sinc function in $\mathbb{R}^{n}$ may be further extended into two larger underlying spaces. The extension to several complex variables involves complexification of each of its real variable. In that case it is a tensor product of the one-dimensional sinc functions, and it has explicit expressions in terms of elementary functions. This case is studied in [3]. The goal of the present paper is to extend it to $\mathbb{R}_{1}^{n}$ in the Clifford analysis setting. The latter extension has no explicit expressions in elementary functions. The definition and the analysis of the extended sinc function are based on the generalized exponential function $e(x, \underline{\xi})$ in $\mathbb{R}_{1}^{n} \times \mathbb{R}^{n}$ (see the following section) extending the classical exponential function $e^{\mathbf{i}\langle\underline{x}, \underline{\xi}\rangle}$ in $\mathbb{R}^{n} \times \mathbb{R}^{n}$, and the corresponding Paley-Wiener Theorem studied in [4].

\section{Preliminaries}

Let $\mathbf{e}_{1}, \ldots, \mathbf{e}_{n}$ be basic elements satisfying $\mathbf{e}_{i} \mathbf{e}_{j}+\mathbf{e}_{j} \mathbf{e}_{i}=-2 \delta_{i j}, i, j=1, \ldots, n$, where $\delta_{i j}$ is the Kronecker $\delta$-function that equals to 1 if $i=j$ and 0 otherwise. Let $\mathbb{R}^{n}=\left\{\underline{x}=x_{1} \mathbf{e}_{1}+\cdots+x_{n} \mathbf{e}_{n}: x_{j} \in \mathbb{R}, j=1, \ldots, n\right\}$ be identified with the usual Euclidean space $\mathbb{R}^{n}$, and $\mathbb{R}_{1}^{n}=\left\{x_{0}+\underline{x}: x_{0} \in \mathbb{R}, \underline{x} \in \mathbb{R}^{n}\right\}$. Elements in $\mathbb{R}_{1}^{n}$ and $\mathbb{R}^{n}$ are called vectors. The real (or complex) Clifford algebra generated by $\mathbf{e}_{1}, \ldots, \mathbf{e}_{n}$, denoted by $\mathbb{R}^{(n)}$, or respectively $\mathbb{C}^{(n)}$, is the associative algebra generated by $\mathbf{e}_{1}, \ldots, \mathbf{e}_{n}$, over the real field $\mathbb{R}$, or respectively the complex field C. A general element in $\mathbb{R}^{(n)}$, for example, is of the form $x=\sum_{S} x_{S} \mathbf{e}_{S}$, where $\mathbf{e}_{S}=\mathbf{e}_{i_{1}} \mathbf{e}_{i_{2}} \cdots \mathbf{e}_{i_{l}}$, and $S$ runs over all the ordered subsets of $\{1, \ldots, n\}$, namely $S=\left\{1 \leq i_{1}<i_{2}<\cdots<i_{l} \leq n\right\}, 0 \leq l \leq n$, where $\mathbf{e}_{\emptyset}=\mathbf{e}_{0}=1$.

The natural inner product between $x$ and $y$ in $\mathbb{C}^{(n)}$, denoted by $\langle x, y\rangle$, is the complex number $\sum_{S} x_{S} \overline{y_{S}}$, where $x=\sum_{S} x_{S} \mathbf{e}_{S}$ and $y=\sum_{S} y_{S} \mathbf{e}_{S}$. The norm associated with this inner product is given by $|x|=\langle x, x\rangle^{\frac{1}{2}}=\left(\sum_{S}\left|x_{S}\right|^{2}\right)^{\frac{1}{2}}$. The conjugate of a vector $x=x_{0}+\underline{x}$ is defined to be $\bar{x}=x_{0}-\underline{x}$. It is easy to verify that for $0 \neq x \in \mathbb{R}_{1}^{n}$ with $x^{-1}=\frac{\bar{x}}{|x|^{2}}$, we have $x^{-1} x=x x^{-1}=1$. The $n$-dimensional unit sphere $\left\{x \in \mathbb{R}_{1}^{n}:|x|=1\right\}$ is denoted by $S^{n}$. We denote by $B(x, r)$ the open ball in $\mathbb{R}_{1}^{n}$ centered at $x$ with radius $r$.

Below, we will study functions defined in $\mathbb{R}^{n}$ or $\mathbb{R}_{1}^{n}$ taking values in $\mathbb{C}^{(n)}$, which have the form $f(x)=\sum_{S} f_{S}(x) \mathbf{e}_{S}$, where $f_{S}$ are complex-valued functions. We will use the Cauchy-Riemann operator $D=D_{0}+\underline{D}$, where $D_{0}=\frac{\partial}{\partial x_{0}}$ and $\underline{D}=\frac{\partial}{\partial x_{1}} \mathbf{e}_{1}+\cdots+\frac{\partial}{\partial x_{n}} \mathbf{e}_{n}$. We also write $D_{0}=\frac{\partial}{\partial x_{0}}=\frac{\partial}{\partial x_{0}} \mathbf{e}_{0}$. We define the "left" and "right" actions of the operators $D$ by

$$
D f=\sum_{i=0}^{n} \sum_{S} \frac{\partial f_{S}}{\partial x_{i}} \mathbf{e}_{i} \mathbf{e}_{S}, \quad f D=\sum_{i=0}^{n} \sum_{S} \frac{\partial f_{S}}{\partial x_{i}} \mathbf{e}_{S} \mathbf{e}_{i},
$$

respectively. 
If in a domain (open and connected) $\Omega$ it holds $D f=0$, then we say that $f$ is left-monogenic; and if $f D=0$, then right-monogenic in $\Omega$. If $f$ is both left- and right-monogenic, then it is monogenic. There hold the corresponding Cauchy's theorem and Cauchy's formula in terms of one-sided monogenic functions for which we refer to [1].

The Fourier transform of functions in $L^{1}\left(\mathbb{R}^{n}\right)$ is defined to be

$$
\mathcal{F}(f)(\underline{\xi})=\int_{\mathbb{R}^{n}} e^{-\mathbf{i}\langle\underline{x}, \underline{\xi}\rangle} f(\underline{x}) d \underline{x},
$$

where $\underline{\xi}=\xi_{1} \mathbf{e}_{1}+\cdots+\xi_{n} \mathbf{e}_{n}$, and the inverse Fourier transform, if applicable, is

$$
\mathcal{F}^{-1}(g)(\underline{x})=\frac{1}{(2 \pi)^{n}} \int_{\mathbb{R}^{n}} e^{\mathbf{i} \underline{x}, \underline{\xi}\rangle} g(\underline{\xi}) d \underline{\xi} .
$$

For a function defined in $\mathbb{R}_{1}^{n}$ we denote $f(\underline{x})=\left(\left.f\right|_{\mathbb{R}^{n}}\right)(\underline{x})$ and $\mathcal{F}(f)=\mathcal{F}\left(\left.f\right|_{\mathbb{R}^{n}}\right)$.

Crucial to this study is an extension of the exponential function $e^{\mathbf{i}\langle\underline{x}, \underline{\xi}\rangle}$. Denote, for $x=x_{0}+\underline{x}$,

$$
e(x, \underline{\xi})=e^{+}(x, \underline{\xi})+e^{-}(x, \underline{\xi})
$$

with

$$
e^{ \pm}(x, \underline{\xi})=e^{\mathbf{i}(\underline{x}, \underline{\xi}\rangle} e^{\mp x_{0}|\underline{\xi}|} \chi_{ \pm}(\underline{\xi}),
$$

where $\chi_{ \pm}(\underline{\xi})=\frac{1}{2}\left(1 \pm \mathbf{i} \frac{\xi}{\mid \underline{\xi}}\right)$. It is easy to verity that $\chi_{ \pm}$enjoy the projection-like properties:

$$
\chi_{-} \chi_{+}=\chi_{+} \chi_{-}=0, \quad \chi_{ \pm}^{2}=\chi_{ \pm}, \quad \chi_{+}+\chi_{-}=1 .
$$

It is easy to verity that $e(x, \underline{\xi})$ is monogenic in $x \in \mathbb{R}_{1}^{n}$ for any fixed $\underline{\xi}$. Generalizations of the exponential function of this kind can be first found in [8] and then in [6] in which $\xi$ is further extended to $\mathbb{C}^{n}$. Generalizations of the exponential function of similar types can also be found in [1,9], and [10].

For $f \in L^{2}\left(\mathbb{R}^{n}\right)$, we will concern the Hardy-space decomposition $f=f^{+}+$ $f^{-}$, where $f^{+}$is the boundary value of a function in the Hardy $H^{2}$-space in the upper half space $\mathbb{R}_{+}^{n+1}=\left\{x=x_{0}+\underline{x}: x_{0}>0, \underline{x} \in \mathbb{R}^{n}\right\}$, and $f^{-}$is the boundary value of a function in the Hardy $H^{2}$-space in the lower half space $\mathbb{R}_{-}^{n+1}=\left\{x=x_{0}+\underline{x}: x_{0}<0, \underline{x} \in \mathbb{R}^{n}\right\}$ (see [6], also [7]). The corresponding Hardy space functions in the upper and lower half spaces, still denoted by $f^{+}$ and $f^{-}$, are given by

$$
f^{ \pm}(x)=\frac{1}{(2 \pi)^{n}} \int_{\mathbb{R}^{n}} \mathcal{F}(f)(\underline{\xi}) e^{ \pm}(x, \underline{\xi}) d \underline{\xi}, \quad \pm x_{0}>0,
$$

respectively. 
In the sequel, in mathematical equalities or inequalities $C$ and $C^{\prime}$ etc. denote positive constants that depend only on the space dimension $n$, and the function-like notation $C(u), C(\sqrt{u})$ denote polynomials in $u$ and $\sqrt{u}$, respectively, with positive coefficients depending only on $n$, and they may vary from one occurrence to another.

\section{Exact interpolation with shannon sampling}

Based on the extension of the exponential function given by (1) and (2) the sinc function in $\mathbb{R}_{1}^{n}$ is defined by

$$
\operatorname{sinc}(x)=\frac{1}{(2 \pi)^{n}} \int_{\mathbb{R}^{n}} e(x, \underline{\xi}) \mathcal{X}_{[-\pi, \pi]^{n}}(\underline{\xi}) d \underline{\xi} .
$$

Due to the decomposition of $e(x, \underline{\xi})$ into $e^{+}(x, \underline{\xi})$ and $e^{-}(x, \underline{\xi})$, the sinc function can be decomposed into a sum of two functions,

$$
\operatorname{sinc}(x)=\operatorname{sinc}^{+}(x)+\operatorname{sinc}^{-}(x),
$$

where

$$
\operatorname{sinc}^{ \pm}(x)=\frac{1}{(2 \pi)^{n}} \int_{\mathbb{R}^{n}} e^{ \pm}(x, \underline{\xi}) \mathcal{X}_{[-\pi, \pi]^{n}}(\underline{\xi}) d \underline{\xi}
$$

and $e^{ \pm}(x, \underline{\xi})$ is defined as in (2). We further have $\operatorname{sinc}^{ \pm}(x)=I_{0}^{ \pm}(x)+\mathbf{i} I_{1}^{ \pm}(x)$ with

$$
\begin{aligned}
& I_{0}^{ \pm}(x)=\frac{1}{2(2 \pi)^{n}} \int_{[-\pi, \pi]^{n}} e^{\mathbf{i}\langle\underline{x}, \underline{\xi}\rangle} e^{\mp x_{0}|\underline{\xi}|} d \underline{\xi} \\
& I_{1}^{ \pm}(x)=\frac{ \pm 1}{2(2 \pi)^{n}} \int_{[-\pi, \pi]^{n}} \frac{\underline{\xi}}{|\underline{\xi}|} e^{\mathbf{i}\langle\underline{x}, \underline{\xi}\rangle} e^{\mp x_{0}|\underline{\xi}|} d \underline{\xi} .
\end{aligned}
$$

For $n=1, z=x+i y \in \mathbb{C}$, the sinc function $\operatorname{sinc}(z)$ satisfies the estimate

$$
|\operatorname{sinc}(z)| \leq \frac{C e^{\pi|y|}}{|z|}
$$

which is consistent with the estimate in the Classical Paley-Wiener theorem. In the above pattern $\operatorname{sinc}(z)$ may be decomposed into $\operatorname{sinc}^{+}(z)$ and $\operatorname{sinc}^{-}(z)$, where $\operatorname{sinc}^{ \pm}(z)$ may be split into the sum of $I_{0}^{ \pm}$and $\mathbf{i} I_{1}^{ \pm}$. Indeed, with $\mathbf{e}_{1}$ replaced 
by $-\mathbf{i}$, we have

$$
\begin{aligned}
\operatorname{sinc}^{ \pm}(z)= & \frac{1}{2 \pi} \int_{-\pi}^{\pi} e^{\mathbf{i} x t} e^{\mp y|t|} \frac{1}{2}\left(1 \pm \mathbf{i} \frac{t e_{1}}{|t|}\right) d t \\
= & I_{0}^{ \pm}(z)+\mathbf{i} I_{1}^{ \pm}(z) \\
= & \left\{\frac{\mp y+e^{\mp \pi y}[x \sin (\pi x) \mp y \cos (\pi x)]}{2 \pi\left(x^{2}+y^{2}\right)}\right\} \\
& +\mathbf{i}\left\{\frac{ \pm x \mp e^{\mp \pi y}[x \cos (\pi x) \pm y \sin (\pi x)]}{2 \pi\left(x^{2}+y^{2}\right)}\right\} .
\end{aligned}
$$

It follows that

$$
\begin{array}{ll}
\left|\operatorname{sinc}^{+}(z)\right| \leq \frac{C\left(1+e^{-\pi y}\right)}{|z|}, & -\infty<y<\infty \\
\left|\operatorname{sinc}^{-}(z)\right| \leq \frac{C\left(1+e^{\pi y}\right)}{|z|}, & -\infty<y<\infty .
\end{array}
$$

In the sequel these estimates will guide us to prove the estimates of $\operatorname{sinc}^{ \pm}(x)$.

For $h>0$ fixed, define the cardinal function of $f$ to be

$$
C(f, h)(x) \equiv \sum_{\underline{k} \in \mathbb{Z}^{n}} f(h \underline{k}) \operatorname{sinc}\left(\frac{x-h \underline{k}}{h}\right),
$$

where from (3),

$$
\operatorname{sinc}\left(\frac{x-h \underline{k}}{h}\right)=\frac{h^{n}}{(2 \pi)^{n}} \int_{\mathbb{R}^{n}} e(x-h \underline{k}, \underline{\xi}) \mathcal{X}_{\left[-\frac{\pi}{h}, \frac{\pi}{h}\right]^{n}}(\underline{\xi}) d \underline{\xi} .
$$

We will concern the interpolation via the cardinal function. The one dimensional cardinal function was discussed in [13]. The context of several complex variables is discussed in [3]. These, together with Theorem 3.4 to be proved below, suggest that the functions whose cardinal function converge to the function itself are characterized by the Paley-Wiener Theorems in the corresponding underlying space.

In [4] we obtain the Paley-Wiener Theorem in the Clifford algebra setting stated as follows.

Theorem 3.1 (Paley-Wiener Theorem). Let $f \in L^{2}\left(\mathbb{R}^{n}\right)$, and $R$ a positive number. Then the following two conditions are equivalent:

(i) $f$ may be right-monogenically extended to the whole $\mathbb{R}_{1}^{n}$, and there exists a constant $C$ such that $|f(x)| \leq C e^{R|x|}$ for all $x \in \mathbb{R}_{1}^{n}$.

(ii) $\operatorname{supp} \mathcal{F}(f) \subset B(0, R)$. 
Moreover, if these conditions hold, then

$$
f(x)=\frac{1}{(2 \pi)^{n}} \int_{B(0, R)} \mathcal{F}(f)(\underline{\xi}) e(x, \underline{\xi}) d \underline{\xi}, \quad x \in \mathbb{R}_{1}^{n} .
$$

A function $f$ in $\mathbb{R}_{1}^{n}$ is said to be of exponential type $R$ if there holds

$$
|f(x)| \leq C e^{R|x|}, \quad x \in \mathbb{R}_{1}^{n}
$$

where $C$ is a positive constant. For any $h>0$, denote by $P W\left(\frac{\pi}{h}\right)$ the totality of the functions $f$, right-monogenic in $\mathbb{R}_{1}^{n},\left.f\right|_{\mathbb{R}^{n}} \in L^{2}\left(\mathbb{R}^{n}\right)$, and of exponential type $\frac{\pi}{h}$.

The Paley-Wiener Theorem 3.1 is a natural generalization of the classical theorem in $\mathbb{C}$. For generalizations to $\mathbb{C}^{n}$, see [12] and [2]. The following theorems characterize the functions in the Paley-Wiener class $P W\left(\frac{\pi}{h}\right)$.

Theorem 3.2. If $f \in P W\left(\frac{\pi}{h}\right)$, then for all $x \in \mathbb{R}_{1}^{n}$,

$$
f(x)=\frac{1}{h^{n}} \int_{\mathbb{R}^{n}} f(\underline{t}) \operatorname{sinc}\left(\frac{x-\underline{t}}{h}\right) d \underline{t} .
$$

Proof. Since $f \in P W\left(\frac{\pi}{h}\right)$, the Paley-Wiener Theorem 3.1 shows that

$$
\begin{aligned}
f(x) & =\frac{1}{(2 \pi)^{n}} \int_{B\left(0, \frac{\pi}{h}\right)} \mathcal{F}(f)(\underline{\xi}) e(x, \underline{\xi}) d \underline{\xi} \\
& =\frac{1}{(2 \pi)^{n}} \int_{\left[-\frac{\pi}{h}, \frac{\pi}{h}\right]^{n}} \mathcal{F}(f)(\underline{\xi}) e(x, \underline{\xi}) d \underline{\xi} \\
& =\frac{1}{(2 \pi)^{n}} \int_{\mathbb{R}^{n}} \mathcal{F}(f)(\underline{\xi}) \mathcal{X}_{\left[-\frac{\pi}{h}, \frac{\pi}{h}\right]^{n}}(\underline{\xi}) e(x, \underline{\xi}) d \underline{\xi} .
\end{aligned}
$$

Using Parseval's Theorem and (5), the above is equal to

$$
\frac{1}{(2 \pi)^{n}} \int_{\mathbb{R}^{n}} f(\underline{t}) \mathcal{F}\left(\mathcal{X}_{\left[-\frac{\pi}{h}, \frac{\pi}{h}\right]^{n}}(\dot{-}) e(x, \underline{-})\right)(\underline{t}) d \underline{t}=\frac{1}{h^{n}} \int_{\mathbb{R}^{n}} f(\underline{t}) \operatorname{sinc}\left(\frac{x-\underline{t}}{h}\right) d \underline{t} .
$$

It is a consequence of (3) and Theorem 3.1 that $\operatorname{sinc}\left(\frac{x}{h}\right)$ belongs to $P W\left(\sqrt{n} \frac{\pi}{h}\right)$. Further examples of functions may be constructed using the following theorem.

Theorem 3.3. If $g \in L^{2}\left(\mathbb{R}^{n}\right)$, then $p \in P W\left(\sqrt{n} \frac{\pi}{h}\right)$, where

$$
p(x)=h^{n} \int_{\mathbb{R}^{n}} g(\underline{t}) \operatorname{sinc}\left(\frac{x-\underline{t}}{h}\right) d \underline{t} .
$$


Proof. Applying Parseval's Theorem to the right-hand side of (6), owing to (5), we have

$$
\begin{aligned}
p(x) & =\frac{h^{n}}{(2 \pi)^{n}} \int_{\mathbb{R}^{n}} \mathcal{F}(g)(\underline{y}) \mathcal{F}\left(\operatorname{sinc}\left(\frac{x-\cdot}{h}\right)\right)(-\underline{y}) d \underline{y} \\
& =\frac{h^{n}}{(2 \pi)^{n}} \int_{\mathbb{R}^{n}} \mathcal{F}(g)(\underline{y})\left[h^{-n} e(x, \underline{y}) \mathcal{X}_{\left[-\frac{\pi}{h}, \frac{\pi}{h}\right]^{n}}(\underline{y})\right] d \underline{y} \\
& =\frac{1}{(2 \pi)^{n}} \int_{\mathbb{R}^{n}} \mathcal{F}(g)(\underline{y}) e(x, \underline{y}) \mathcal{X}_{\left[-\frac{\pi}{h}, \frac{\pi}{h}\right]^{n}}(\underline{y}) d \underline{y} .
\end{aligned}
$$

This shows that $\operatorname{supp} \mathcal{F}(p) \subset\left[-\frac{\pi}{h}, \frac{\pi}{h}\right]^{n} \subset B\left(0, \sqrt{n} \frac{\pi}{h}\right)$. From Paley-Wiener Theorem 3.1, we have $p \in P W\left(\sqrt{n} \frac{\pi}{h}\right)$.

As main result, the exact sinc interpolation of functions in $P W\left(\frac{\pi}{h}\right)$ is given by the following

Theorem 3.4. If $f \in P W\left(\frac{\pi}{h}\right)$, then for all $x \in \mathbb{R}_{1}^{n}$,

$$
f(x)=C(f, h)(x)=\sum_{\underline{k} \in \mathbb{Z}^{n}} f(h \underline{k}) \operatorname{sinc}\left(\frac{x-h \underline{k}}{h}\right),
$$

where the series on the right-hand side is absolutely and uniformly convergent. Moreover,

$$
f(h \underline{k})=\frac{1}{h^{n}} \int_{\mathbb{R}^{n}} f(\underline{t}) \operatorname{sinc}\left(\frac{\underline{t}-h \underline{k}}{h}\right) d \underline{t} .
$$

Proof. The relation $f(h \underline{k})=\frac{1}{h^{n}} \int_{\mathbb{R}^{n}} f(\underline{t}) \operatorname{sinc}\left(\frac{\underline{t}-h \underline{k}}{h}\right) d \underline{t}$ follows from Theorem 3.2.

To show (7), we need to use the fact that $\sum_{\underline{k} \in \mathbb{Z}^{n}}|f(h \underline{k})|^{2}$ is finite. We show this by proving,

$$
\sum_{\underline{k} \in \mathbb{Z}^{n}}|f(h \underline{k})|^{2}=\int_{\mathbb{R}^{n}}|f(\underline{t})|^{2} d \underline{t} .
$$

In fact, from Paley-Wiener Theorem 3.1,

$$
f(\underline{t})=\frac{1}{(2 \pi)^{n}} \int_{B\left(0, \frac{\pi}{h}\right)} \mathcal{F}(f)(\underline{x}) e^{\mathbf{i}\langle\underline{x}, \underline{t}\rangle} d \underline{x}=\frac{1}{(2 \pi)^{n}} \int_{\left[-\frac{\pi}{h}, \frac{\pi}{h}\right]^{n}} \mathcal{F}(f)(\underline{x}) e^{\mathbf{i}\langle\underline{x}, \underline{t}\rangle} d \underline{x} .
$$

The Fourier coefficients of $\mathcal{F}(f)$ in the cube $\left[-\frac{\pi}{h}, \frac{\pi}{h}\right]^{n}$ are

$$
c_{\underline{k}}=h^{n} f(h \underline{k})=\frac{1}{(2 R)^{n}} \int_{[-R, R]^{n}} \mathcal{F}(f)(\underline{x}) e^{\mathbf{i} \pi \frac{\langle\underline{x}, k\rangle}{R}} d \underline{x},
$$


where $R=\frac{\pi}{h}$. The Plancherel Theorem gives

$$
\int_{[-R, R]^{n}}|\mathcal{F}(f)(\underline{x})|^{2} d \underline{x}=(2 R)^{n} \sum_{\underline{k} \in \mathbb{Z}^{n}}\left|c_{\underline{k}}\right|^{2}=(2 \pi)^{n} \sum_{\underline{k} \in \mathbb{Z}^{n}}|f(h \underline{k})|^{2} .
$$

Taking into account that $\operatorname{supp}(\mathcal{F}(f)) \subset[-R, R]^{n}$, Parseval's Theorem on $L^{2}$ functions in $\mathbb{R}^{n}$ gives

$$
\int_{[-R, R]^{n}}|\mathcal{F}(f)(\underline{x})|^{2} d \underline{x}=\int_{\mathbb{R}^{n}}|\mathcal{F}(f)(\underline{x})|^{2} d \underline{x}=(2 \pi)^{n} \int_{\mathbb{R}^{n}}|f(\underline{t})|^{2} d \underline{t} .
$$

Putting these equalities together, we obtain (8).

We next show that in the uniform convergence sense,

$$
f(\underline{x})=C(f, h)(\underline{x})=\sum_{\underline{k} \in \mathbb{Z}^{n}} f(h \underline{k}) \operatorname{sinc}\left(\frac{\underline{x}-h \underline{k}}{h}\right) \quad \forall \underline{x} \in \mathbb{R}^{n} .
$$

Let $\phi_{\epsilon}(\underline{x})$ be an infinitely differentiable function such that $\operatorname{supp}\left(\phi_{\epsilon}\right) \subset\left[-\frac{\pi}{h}, \frac{\pi}{h}\right]^{n}$, $\left|\phi_{\epsilon}\right| \leq 1$ and $\phi_{\epsilon} \rightarrow \chi_{\left[-\frac{\pi}{h}, \frac{\pi}{h}\right]^{n}}$ a.e. as $\epsilon \rightarrow 0$. Define

$$
I_{\epsilon} f(\underline{x})=\frac{1}{(2 \pi)^{n}} \int_{\left[-\frac{\pi}{h}, \frac{\pi}{h}\right]^{n}} \mathcal{F}(f)(\underline{t}) \phi_{\epsilon}(\underline{t}) e^{\mathbf{i}(\underline{\underline{t}, \underline{x}\rangle} d \underline{t} .}
$$

For any fixed $\epsilon>0$ and $\underline{x} \in \mathbb{R}^{n}$, expanding $\phi_{\epsilon}(\underline{t}) e^{\mathbf{i}\{\underline{\langle} \underline{\underline{x}}\rangle}$ on the cube $\left[-\frac{\pi}{h}, \frac{\pi}{h}\right]^{n}$ into its multiple Fourier series in $n$-variables, we have

$$
\phi_{\epsilon}(\underline{t}) e^{\mathbf{i}\langle\underline{t}, \underline{x}\rangle}=\sum_{\underline{k} \in \mathbb{Z}^{n}} e^{\mathrm{i} h\langle\underline{k}, \underline{t}\rangle} S_{\epsilon}(\underline{k}, \underline{x}),
$$

where

$$
S_{\epsilon}(\underline{k}, \underline{x})=\frac{1}{(2 R)^{n}} \int_{[-R, R]^{n}} e^{-\mathbf{i} \pi \frac{\langle t, k\rangle}{R}}\left[\phi_{\epsilon}(\underline{t}) e^{\mathrm{i}\langle\underline{t}, \underline{x}\rangle}\right] d \underline{t}
$$

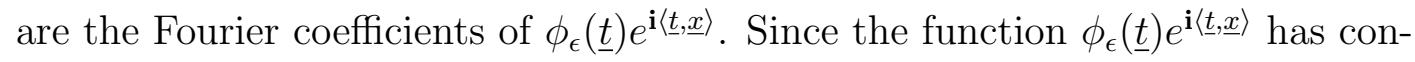
tinuous first and second derivatives and vanishes on the boundary of the cube $\left[-\frac{\pi}{h}, \frac{\pi}{h}\right]^{n}$, a routine argument based on integration by parts shows that

$$
\left|S_{\epsilon}(\underline{k}, \underline{x})\right| \leq \frac{C(\epsilon, \underline{x})}{\underline{k}^{2}} \quad \forall \underline{x} \in \mathbb{R}^{n} .
$$

So the series (11) is absolutely convergent, uniformly in $\underline{t}$ for any fixed $\epsilon$ and $\underline{x}$.

Inserting the series (11) into (10) and exchanging the order of integration and summation, justified by the uniform convergence, we have

$$
I_{\epsilon} f(\underline{x})=\sum_{\underline{k} \in \mathbb{Z}^{n}}\left[\frac{1}{(2 \pi)^{n}} \int_{\left[-\frac{\pi}{h}, \frac{\pi}{h}\right]^{n}} \mathcal{F}(f)(\underline{t}) e^{\mathrm{i} h\langle\underline{k}, \underline{t}\rangle} d \underline{\underline{t}}\right] S_{\epsilon}(\underline{k}, \underline{x}) .
$$


Inserting the expression

$$
f(h \underline{k})=\frac{1}{(2 \pi)^{n}} \int_{B\left(0, \frac{\pi}{h}\right)} \mathcal{F}(f)(\underline{t}) e^{\mathrm{i} h\langle\underline{k}, \underline{t}\rangle} d \underline{t}=\frac{1}{(2 \pi)^{n}} \int_{\left[-\frac{\pi}{h}, \frac{\pi}{h}\right]^{n}} \mathcal{F}(f)(\underline{t}) e^{\mathrm{i} h\langle\underline{k}, \underline{t}\rangle} d \underline{t},
$$

(12) becomes

$$
I_{\epsilon} f(\underline{x})=\sum_{\underline{k} \in \mathbb{Z}^{n}} f(h \underline{k}) S_{\epsilon}(\underline{k}, \underline{x}) .
$$

On taking the limit $\epsilon \rightarrow 0$ to (13), first consider its left-hand side. Since $\mathcal{F}(f) \in L^{2}\left(B\left(0, \frac{\pi}{h}\right)\right) \subset L^{1}\left(B\left(0, \frac{\pi}{h}\right)\right)$, we have

$$
\left|\mathcal{F}(f)(\underline{t}) \phi_{\epsilon}(\underline{t}) e^{\mathbf{i}\langle\underline{t}, \underline{x}\rangle}\right| \leq\left|\mathcal{F}(f)(\underline{t}) e^{\mathbf{i}\langle\underline{t}, \underline{x}\rangle}\right| \in L^{1}\left(\left[-\frac{\pi}{h}, \frac{\pi}{h}\right]^{n}\right) .
$$

Moreover, by the definition of $\phi_{\epsilon}$, we have

$$
\lim _{\epsilon \rightarrow 0} \mathcal{F}(f)(\underline{t}) \phi_{\epsilon}(\underline{x}) e^{\mathbf{i}\langle\underline{t}, \underline{x}\rangle}=\mathcal{F}(f)(\underline{t}) e^{\mathbf{i}\langle\underline{t}, \underline{x}\rangle} .
$$

Using Legesgue's Dominated Convergence Theorem, the left hand side of (13), by recalling (10), tends to

$$
\frac{1}{(2 \pi)^{n}} \int_{\left[-\frac{\pi}{h}, \frac{\pi}{h}\right]^{n}} \mathcal{F}(f)(\underline{t}) e^{\mathbf{i}\langle\underline{t}, \underline{x}\rangle} d \underline{t}=f(\underline{x}),
$$

where the last equality is a consequence of the Paley-Wiener Theorem 3.1.

Next, consider the series on the right-hand side of (13). For any positive number $M$, using the Cauchy-Schwarz inequality, we have

$$
\left|\sum_{|\underline{k}|>M} f(h \underline{k}) S_{\epsilon}(\underline{k}, \underline{x})\right| \leq\left(\sum_{|\underline{k}|>M}|f(h \underline{k})|^{2}\right)^{\frac{1}{2}}\left(\sum_{|\underline{k}|>M}\left|S_{\epsilon}(\underline{k}, \underline{x})\right|^{2}\right)^{\frac{1}{2}} .
$$

Note that the function $\phi_{\epsilon}(\underline{t}) e^{\mathbf{i}\langle\underline{t}, \underline{x}\rangle} \in L^{2}\left(\left[-\frac{\pi}{h}, \frac{\pi}{h}\right]^{n}\right)$. The Bessel inequality gives

$$
\begin{aligned}
\left(\sum_{|\underline{k}|>M}\left|S_{\epsilon}(\underline{k}, \underline{x})\right|^{2}\right)^{\frac{1}{2}} & \leq\left(\frac{h}{2 \pi}\right)^{\frac{n}{2}}\left\|\phi_{\epsilon}(\underline{t}) e^{\mathbf{i}\langle\underline{t}, \underline{x}\rangle}\right\|_{L^{2}\left(\left[-\frac{\pi}{h}, \frac{\pi}{h}\right]^{n}\right)} \\
& \leq\left(\frac{h}{2 \pi}\right)^{\frac{n}{2}}\left\|e^{\mathbf{i}\langle\underline{t}, \underline{x}\rangle}\right\|_{L^{2}\left(\left[-\frac{\pi}{h}, \frac{\pi}{h}\right]^{n}\right)} \\
& \leq C<\infty .
\end{aligned}
$$

Owing to this estimate and (8), the series in (13) is convergent uniformly in $\epsilon$ and $\underline{x}$. Now take limit $\epsilon \rightarrow 0$ on the right-hand-side of (13). Since we can 
exchange the limit procedure with the summation, that is based on the just proved uniform convergence in $\epsilon$, the limit procedure can be passed onto each term $S_{\epsilon}(\underline{k}, \underline{x})$. By invoking the relation

$$
\lim _{\epsilon \rightarrow 0} S_{\epsilon}(\underline{k}, \underline{x})=\operatorname{sinc}\left(\frac{\underline{x}-h \underline{k}}{h}\right),
$$

we obtain (9).

The relation (9) shows that, when restricted to $\mathbb{R}^{n}$, the left-hand-side and the right-hand side of $(7)$, viz. $f(\underline{x})$ and $C(f, h)(\underline{x})$, respectively, coincide with each other. Now $f$ is right-monogenic in $\mathbb{R}_{1}^{n}$, if we can show that $C(f, h)$ is also right-monogenic in $\mathbb{R}_{1}^{n}$, then the corresponding uniqueness theorem in Clifford analysis (see [1]) will allow us to conclude (7), and thus complete the proof. Below, we will devote ourselves to proving the uniform convergence of the series $C(f, h)(x)$, and thus the monogenicity of the sum $C(f, h)(x)$.

The rest of the proof depends on the following technical lemmas.

Lemma 3.5. It holds:

$$
|\operatorname{sinc}(x)| \leq \frac{C\left(\left|x_{0}\right|\right) e^{\sqrt{n} \pi\left|x_{0}\right|}}{\prod_{j=1}^{n}\left(1+\left|x_{j}\right|\right)} \quad \forall x \in \mathbb{R}_{1}^{n},
$$

where $C\left(\left|x_{0}\right|\right)$ is a polynomial of $\left|x_{0}\right|$.

Proof. Using the decomposition of sinc into sinc ${ }^{+}$and sinc ${ }^{-}$as cited in (4), it suffices to show the estimate

$$
\left|\operatorname{sinc}^{ \pm}(x)\right| \leq \frac{C\left(\left|x_{0}\right|\right) e^{\sqrt{n} \pi\left|x_{0}\right|}}{\prod_{j=1}^{n}\left(1+\left|x_{j}\right|\right)} \quad \forall x \in \mathbb{R}_{1}^{n} .
$$

We only need to prove the last inequality for $\operatorname{sinc}^{+}$as the proof for $\operatorname{sinc}^{-}$is similar. By definition

$$
\operatorname{sinc}^{+}(x)=\frac{1}{(2 \pi)^{n}} \int_{[-\pi, \pi]^{n}} e^{\mathbf{i}\langle\underline{\underline{\xi}} \underline{\xi}\rangle} e^{-x_{0}|\underline{\xi}|} \frac{1}{2}\left(1+\mathbf{i} \frac{\underline{\xi}}{|\underline{\xi}|}\right) d \underline{\xi}=I_{0}^{+}(x)+\mathbf{i} I_{1}^{+}(x),
$$

where

$$
\begin{aligned}
& I_{0}^{+}(x)=\frac{1}{2(2 \pi)^{n}} \int_{[-\pi, \pi]^{n}} e^{\mathbf{i}\langle\underline{x}, \underline{\xi}\rangle} e^{-x_{0}|\underline{\xi}|} d \underline{\xi} \\
& I_{1}^{+}(x)=\frac{1}{2(2 \pi)^{n}} \int_{[-\pi, \pi]^{n}} e^{\mathbf{i}|\underline{x}, \underline{\xi}\rangle} e^{-x_{0}|\underline{\xi}|} \frac{\underline{\xi}}{|\underline{\xi}|} d \underline{\xi} .
\end{aligned}
$$

We first show that

$$
\left|I_{0}^{+}(x)\right| \leq \frac{C\left(\left|x_{0}\right|\right) e^{\sqrt{n} \pi\left|x_{0}\right|}}{\prod_{j=1}^{n}\left(1+\left|x_{j}\right|\right)} \quad \forall x \in \mathbb{R}_{1}^{n}
$$


Consider

$$
\left(1+\left|x_{1}\right|\right) \cdots\left(1+\left|x_{n}\right|\right)\left|I_{0}^{+}(x)\right|=\sum_{0 \leq k \leq n}\left|x_{i_{1}} \cdots x_{i_{k}} I_{0}^{+}(x)\right|,
$$

where for $1 \leq k \leq n, 1 \leq i_{1}<\ldots<i_{k} \leq n$, and for $k=0$ the corresponding term is $\left|I_{0}^{+}(x)\right|$. Note that

$$
\left|x_{i_{1}} \cdots x_{i_{k}} I_{0}^{+}(x)\right|=C\left|\int_{[-\pi, \pi]^{n}} \frac{\partial^{k}}{\partial \xi_{i_{1}} \ldots \partial \xi_{i_{k}}}\left(e^{\mathbf{i}\langle\underline{x}, \underline{\xi}\rangle}\right) e^{-x_{0}|\underline{\xi}|} d \underline{\xi}\right| .
$$

Applying integration by parts $k$ times with respect to $d \xi_{i_{1}}, \ldots, d \xi_{i_{k}}$, respectively, the above is dominated by

$$
C\left(\left|x_{0}\right|\right) e^{\sqrt{n} \pi\left|x_{0}\right|} \int_{[-\pi, \pi]^{n}} \sum_{l=1}^{k} \frac{1}{|\underline{\xi}|^{l-1}} d \underline{\xi} \leq C\left(\left|x_{0}\right|\right) e^{\sqrt{n} \pi\left|x_{0}\right|}, \quad 0 \leq k \leq n .
$$

So, (15) follows.

Now we show that

$$
\left|I_{1}^{+}(x)\right| \leq \frac{C\left(\left|x_{0}\right|\right) e^{\sqrt{n} \pi\left|x_{0}\right|}}{\prod_{i=1}^{n}\left(1+\left|x_{i}\right|\right)}, \quad \forall x \in \mathbb{R}_{1}^{n} .
$$

The strategy is to find appropriate estimates for

$$
J(x)=\frac{\partial}{\partial x_{0}} I_{1}^{+}(x)=-C \int_{[-\pi, \pi]^{n}} e^{\mathbf{i}\langle\underline{x}, \underline{\xi}\rangle} e^{-x_{0}|\underline{\xi}|} \underline{\xi} d \underline{\xi}
$$

and then, using the relation $I_{1}^{+}(x)=-\int_{x_{0}}^{\infty} J(t+\underline{x}) d t$ get the estimate for $I_{1}^{+}(x)$. Using the same method as in deducing the inequality (15) we have

$$
|J(x)| \leq \frac{C\left(\left|x_{0}\right|\right) e^{\sqrt{n} \pi\left|x_{0}\right|}}{\prod_{i=1}^{n}\left(1+\left|x_{i}\right|\right)} \quad \forall x \in \mathbb{R}_{1}^{n} .
$$

This estimate is for general $x \in \mathbb{R}_{1}^{n}$, and in particular more exact for $x_{0}<0$, which will play a role latter. For positive $x_{0}$, however, we need an estimate that guarantees the integrability of $J(t+\underline{x})$ at the infinity.

Now we show that for $x_{0}>1$,

$$
|J(x)| \leq \frac{C}{x_{0} \prod_{i=1}^{n}\left(x_{0}+\left|x_{i}\right|\right)}, \quad \forall \underline{x} \in \mathbb{R}^{n},
$$

where $C$ is a constant depending only on the space dimension $n$. Below, we restrict ourselves to $n=2$. For $n>2$ the proof is similar. As defined, for $n=2$,

$$
J(x)=-C \int_{[-\pi, \pi]^{2}} e^{\mathbf{i}\langle\underline{x}, \underline{\xi}\rangle} e^{-x_{0}|\underline{\xi}|} \underline{\xi} d \underline{\xi} .
$$


Applying the identity

$$
e^{-\beta}=\frac{1}{\sqrt{\pi}} \int_{0}^{\infty} \frac{e^{-u}}{\sqrt{u}} e^{-\frac{\beta^{2}}{4 u}} d u
$$

(see [12]) for $\beta=x_{0}|\xi|$ to the exponential function in the integrand of $J(x)$, we have

$$
\begin{aligned}
J(x)= & \frac{-C}{\sqrt{\pi}} \int_{0}^{\infty} \frac{e^{-u}}{\sqrt{u}} \int_{-\pi}^{\pi} \int_{-\pi}^{\pi} \xi_{1} e^{\mathbf{i}\langle\underline{x}, \underline{\xi}\rangle} e^{-\frac{x_{0}^{2} \xi_{1}^{2}+x_{0}^{2} \xi_{2}^{2}}{4 u}} d \xi_{1} d \xi_{2} d u \\
= & \frac{-4 C}{\sqrt{\pi}} \int_{0}^{\infty} \frac{e^{-u}}{\sqrt{u}}\left(\int_{0}^{\pi} \xi_{1} \sin \left(x_{1} \xi_{1}\right) e^{-\frac{x_{0}^{2} \xi_{1}^{2}}{4 u}} d \xi_{1}\right) \\
& \times\left(\int_{0}^{\pi} \cos \left(x_{2} \xi_{2}\right) e^{-\frac{x_{0}^{2} \xi_{2}^{2}}{4 u}} d \xi_{2}\right) d u .
\end{aligned}
$$

First study the last integral. On one hand,

$$
\left|\int_{0}^{\pi} \cos \left(x_{2} \xi_{2}\right) e^{-\frac{x_{0}^{2} \xi_{2}^{2}}{4 u}} d \xi_{2}\right| \leq \int_{0}^{\infty} e^{-\frac{x_{0}^{2} \xi_{2}^{2}}{4 u}} d \xi_{2}=\frac{C \sqrt{u}}{x_{0}} .
$$

On the other hand, the Second Mean Value Theorem of integration gives

$$
\left|\int_{0}^{\pi} \cos \left(x_{2} \xi_{2}\right) e^{-\frac{x_{0}^{2} \xi_{2}^{2}}{4 u}} d \xi_{2}\right|=\left|\int_{0}^{\eta} \cos \left(x_{2} \xi_{2}\right) d \xi_{2}\right| \leq \frac{C^{\prime}}{\left|x_{2}\right|} .
$$

The last two estimates may be combined together to obtain

$$
\left|\int_{0}^{\pi} \cos \left(x_{2} \xi_{2}\right) e^{-x_{0}^{2} \xi_{2}^{2}} d \xi_{2}\right| \leq \frac{C \sqrt{u}+C^{\prime}}{x_{0}+\left|x_{2}\right|}=\frac{C(\sqrt{u})}{x_{0}+\left|x_{2}\right|}
$$

where $C(\sqrt{u})$ is a polynomial in $\sqrt{u}$.

Next, we have, similarly,

$$
\left|\int_{0}^{\pi} \xi_{1} \sin \left(x_{1} \xi_{1}\right) e^{-\frac{x_{0}^{2} \xi_{1}^{2}}{4 u}} d \xi_{1}\right| \leq \int_{0}^{\infty} \xi_{1} e^{-\frac{x_{0}^{2} \xi_{1}^{2}}{4 u}} d \xi_{1} \leq \frac{C u}{x_{0}^{2}} .
$$

To get an estimate in terms of $\left|x_{1}\right|$, consider the function $f(t)=t e^{-x_{0}^{2} t^{2} / 4 u}$. Since $f^{\prime}(t)=\left(1-\frac{2 t^{2} x_{0}^{2}}{4 u}\right) e^{-x_{0}^{2} t^{2} / 4 u}$, the maximum of $f(t)$ is $\frac{\sqrt{2 u}}{x_{0}} e^{-\frac{1}{2}}$, occurring at $\frac{\sqrt{2 u}}{x_{0}}$.

We discuss two cases. First assume $t_{0}=\frac{\sqrt{2 u}}{x_{0}} \leq \pi$, that implies $u \leq \frac{\pi^{2} x_{0}^{2}}{2}$. From the Second Mean Value Theorem of integration, we have

$$
\begin{aligned}
\int_{0}^{\pi} \xi_{1} \sin \left(x_{1} \xi_{1}\right) e^{-\frac{x_{0}^{2} \xi_{1}^{2}}{4 u}} d \xi_{1} \\
=\int_{0}^{t_{0}} \xi_{1} \sin \left(x_{1} \xi_{1}\right) e^{-\frac{x_{0}^{2} \xi_{1}^{2}}{4 u}} d \xi_{1}+\int_{t_{0}}^{\pi} \xi_{1} \sin \left(x_{1} \xi_{1}\right) e^{-\frac{x_{0}^{2} \xi_{1}^{2}}{4 u}} d \xi_{1} \\
=\frac{\sqrt{2 u} e^{-\frac{1}{2}}}{x_{0}} \int_{\eta_{1}}^{t_{0}} \sin \left(x_{1} \xi_{1}\right) d \xi_{1}+\frac{\sqrt{2 u} e^{-\frac{1}{2}}}{x_{0}} \int_{t_{0}}^{\eta_{2}} \sin \left(x_{1} \xi_{1}\right) d \xi_{1} .
\end{aligned}
$$


Since

$$
\left|\int_{\eta}^{t_{0}} \sin \left(x_{1} \xi_{1}\right) d \xi_{1}\right|+\left|\int_{t_{0}}^{\eta_{2}} \sin \left(x_{1} \xi_{1}\right) d \xi_{1}\right| \leq \frac{C}{\left|x_{1}\right|}
$$

we obtain

$$
\left|\int_{0}^{\pi} \xi_{1} \sin \left(x_{1} \xi_{1}\right) e^{-\frac{x_{0}^{2} \xi_{1}^{2}}{4 u}} d \xi_{1}\right| \leq \frac{C \sqrt{u}}{x_{0}\left|x_{1}\right|}
$$

Combining this with (21), we obtain

$$
\left|\int_{0}^{\pi} \xi_{1} \sin \left(x_{1} \xi_{1}\right) e^{-\frac{x_{0}^{2} \xi_{1}^{2}}{4 u}} d \xi_{1}\right| \leq \frac{C(\sqrt{u})}{x_{0}\left(x_{0}+\left|x_{1}\right|\right)}
$$

where $C(\sqrt{u})$ is a polynomial in $\sqrt{u}$.

Second, assume $t_{0}=\frac{\sqrt{2 u}}{x_{0}}>\pi$, that implies $u>\frac{\pi^{2} x_{0}^{2}}{2}$. Then $f(t)$ is increasing in the whole range $[0, \pi]$. The Second Mean Value Theorem of integration and the estimate $e^{-t}<\frac{1}{t}, t>0$, then imply

$$
\left|\int_{0}^{\pi} \xi_{1} \sin \left(x_{1} \xi_{1}\right) e^{-\frac{x_{0}^{2} \xi_{1}^{2}}{4 u}} d \xi_{1}\right|=\pi e^{-\frac{x_{0}^{2} \pi^{2}}{4 u}}\left|\int_{\eta}^{\pi} \sin \left(x_{1} \xi_{1}\right) d \xi_{1}\right| \leq \frac{C e^{-\frac{x_{0}^{2} \pi^{2}}{4 u}}}{\left|x_{1}\right|} \leq \frac{C u}{x_{0}^{2}\left|x_{1}\right|} .
$$

Combining this with (21), due to $x_{0}>1$, we have

$$
\left|\int_{0}^{\pi} \xi_{1} \sin \left(x_{1} \xi_{1}\right) e^{-\frac{x_{0}^{2} \xi_{1}^{2}}{4 u}} d \xi_{1}\right| \leq \frac{C u}{x_{0}^{2}\left(1+\left|x_{1}\right|\right)} \leq \frac{C u}{x_{0}\left(x_{0}+\left|x_{1}\right|\right)} .
$$

By invoking (20), (22) and (23), the integral expression for $J(x)$ given in (19) is dominated by

$$
\int_{0}^{\infty} \frac{e^{-u}}{\sqrt{u}}\left[\frac{C(\sqrt{u})+C u}{x_{0}\left(x_{0}+\left|x_{1}\right|\right)}\right]\left[\frac{C(\sqrt{u})}{x_{0}+\left|x_{2}\right|}\right] d u \leq \frac{C}{x_{0}\left(x_{0}+\left|x_{1}\right|\right)\left(x_{0}+\left|x_{2}\right|\right)},
$$

as desired in (18).

Now for any $x_{0}$, using the estimates (17) and (18) for $n=2$, we have

$$
\begin{aligned}
\left|I_{1}^{+}(x)\right|= & \left|-\int_{x_{0}}^{\infty} J(t+\underline{x}) d t\right| \\
\leq & \left|\int_{x_{0}}^{1} J(t+\underline{x}) d t\right|+\int_{1}^{\infty}|J(t+\underline{x})| d t \\
\leq & \frac{1}{\left(1+\left|x_{1}\right|\right)\left(1+\left|x_{2}\right|\right)}\left|\int_{x_{0}}^{1} C(t) e^{\sqrt{2} \pi|t|} d t\right| \\
& +C \int_{1}^{\infty} \frac{1}{t\left(t+\left|x_{1}\right|\right)\left(t+\left|x_{2}\right|\right)} d t \\
\leq & \frac{C\left(\left|x_{0}\right|\right) e^{\sqrt{2} \pi\left|x_{0}\right|}}{\left(1+\left|x_{1}\right|\right)\left(1+\left|x_{2}\right|\right)}+C \int_{1}^{\infty} \frac{1}{t\left(t+\left|x_{1}\right|\right)\left(t+\left|x_{2}\right|\right)} d t .
\end{aligned}
$$


Next we show that

$$
\int_{1}^{\infty} \frac{1}{t\left(t+\left|x_{1}\right|\right)\left(t+\left|x_{2}\right|\right)} d t \leq \frac{C}{\left(1+\left|x_{1}\right|\right)\left(1+\left|x_{2}\right|\right)}
$$

The evaluation of the last integral is

$$
\begin{aligned}
\int_{1}^{\infty} \frac{1}{t\left(t+\left|x_{1}\right|\right)\left(t+\left|x_{2}\right|\right)} d t= & -\frac{\ln \left(1+\left|x_{1}\right|\right)}{\left|x_{1}\right|\left(\left|x_{1}\right|-\left|x_{2}\right|\right)}+\frac{\ln \left(1+\left|x_{2}\right|\right)}{\left|x_{2}\right|\left(\left|x_{1}\right|-\left|x_{2}\right|\right)} \\
= & \frac{1}{\left|x_{1}\right|\left|x_{2}\right|}\left[-\frac{\ln \left(1+\left|x_{1}\right|\right)^{\left(1+\left|x_{2}\right|\right)}-\ln \left(1+\left|x_{2}\right|\right)^{\left(1+\left|x_{1}\right|\right)}}{\left(1+\left|x_{1}\right|\right)-\left(1+\left|x_{2}\right|\right)}\right. \\
& \left.+\frac{\ln \left(1+\left|x_{1}\right|\right)-\ln \left(1+\left|x_{2}\right|\right)}{\left(1+\left|x_{1}\right|\right)-\left(1+\left|x_{2}\right|\right)}\right] .
\end{aligned}
$$

Denoting $y_{1}=1+\left|x_{1}\right|$ and $y_{2}=1+\left|x_{2}\right|$, the above is equal to

$$
\frac{1}{\left|x_{1}\right|\left|x_{2}\right|}\left[-\frac{\ln y_{1}^{y_{2}}-\ln y_{2}^{y_{1}}}{y_{1}-y_{2}}+\frac{\ln y_{1}-\ln y_{2}}{y_{1}-y_{2}}\right] \text {. }
$$

Since for any $y_{1}>1$ and $y_{2}>1$, the quantities $\left(\ln y_{1}^{y_{2}}-\ln y_{2}^{y_{1}}\right) /\left(y_{1}-y_{2}\right)$ and $\left(\ln y_{1}-\ln y_{2}\right) /\left(y_{1}-y_{2}\right)$ both are bounded, we obtain

$$
\int_{1}^{\infty} \frac{1}{t\left(t+\left|x_{1}\right|\right)\left(t+\left|x_{2}\right|\right)} d t \leq \frac{C}{\left|x_{1}\right|\left|x_{2}\right|}
$$

Since obviously

$$
\int_{1}^{\infty} \frac{1}{t\left(t+\left|x_{1}\right|\right)\left(t+\left|x_{2}\right|\right)} d t \leq \frac{1}{1+\left|x_{i}\right|} \int_{1}^{\infty} \frac{1}{t^{2}} d t \leq \frac{C}{1+\left|x_{i}\right|}, \quad i=1,2,
$$

we conclude

$$
\int_{1}^{\infty} \frac{1}{t\left(t+\left|x_{1}\right|\right)\left(t+\left|x_{2}\right|\right)} d t \leq \frac{C}{1+\left|x_{1}\right|+\left|x_{2}\right|+\left|x_{1}\right|\left|x_{2}\right|} \leq \frac{C}{\left(1+\left|x_{1}\right|\right)\left(1+\left|x_{2}\right|\right)}
$$

as desired in (25).

Using the estimate (25) in (24), we have

$$
\left|I_{1}^{+}(x)\right| \leq \frac{C\left(\left|x_{0}\right|\right) e^{\sqrt{2} \pi\left|x_{0}\right|}}{\left(1+\left|x_{1}\right|\right)\left(1+\left|x_{2}\right|\right)} \quad \forall x \in \mathbb{R}_{1}^{n} .
$$

Combining (15) and (26), we obtain

$$
\left|\operatorname{sinc}^{+}(x)\right| \leq \frac{C\left(\left|x_{0}\right|\right) e^{\sqrt{2} \pi\left|x_{0}\right|}}{\left(1+\left|x_{1}\right|\right)\left(1+\left|x_{2}\right|\right)} \quad \forall x \in \mathbb{R}_{1}^{n} .
$$


The above proof can be generalized to arbitrary dimension number $n$ and shows that

$$
\left|\operatorname{sinc}^{+}(x)\right| \leq \frac{C\left(\left|x_{0}\right|\right) e^{\sqrt{n} \pi\left|x_{0}\right|}}{\prod_{i=1}^{n}\left(1+\left|x_{i}\right|\right)} \quad \forall x \in \mathbb{R}_{1}^{n} .
$$

This completes the proof of Lemma 3.5.

Now we show

Lemma 3.6. The cardinal function $C(f, h)(x)$ is uniformly convergent.

Proof. Recall that

$$
C(f, h)(x)=\sum_{\underline{k} \in \mathbf{Z}^{n}} f(h \underline{k}) \operatorname{sinc}\left(\frac{x-h \underline{k}}{h}\right) .
$$

For any positive number $M$, using the Cauchy-Schwarz inequality,

$$
\begin{aligned}
& \left|\sum_{|\underline{k}|>M} f(h \underline{k}) \operatorname{sinc}\left(\frac{x-h \underline{k}}{h}\right)\right| \\
& \leq\left(\sum_{|\underline{k}|>M}|f(h \underline{k})|^{2}\right)^{\frac{1}{2}}\left(\sum_{|\underline{k}|>M}\left|\operatorname{sinc}\left(\frac{x-h \underline{k}}{h}\right)\right|^{2}\right)^{\frac{1}{2}} .
\end{aligned}
$$

Applying Lemma 3.5 to the square of the second factor, we have

$$
\begin{aligned}
\sum_{|\underline{k}|>M} \mid \operatorname{sinc} & \left.\left(\frac{x-h \underline{k}}{h}\right)\right|^{2} \\
& \leq C\left(\left|\frac{x_{0}}{h}\right|\right) e^{\sqrt{n} \pi \frac{\left|x_{0}\right|}{h}} \sum_{|\underline{\mid k}|>M} \frac{1}{\prod_{j=1}^{n}\left[1+\left|\frac{\left(x_{j}-h k_{j}\right)}{h}\right|\right]^{2}} .
\end{aligned}
$$

For a fixed $h>0$, the last sum is uniformly bounded for all $\underline{x} \in \mathbb{R}^{n}$ and $M>0$, and the factor in front of it, viz. $C\left(\left|\frac{x_{0}}{h}\right|\right) e^{\sqrt{n} \pi\left|x_{0}\right| / h}$, is uniformly bounded in any bounded neighborhood of $x_{0}$. The first factor of (27), owing to (8), tends to zero as $M$ goes to infinity. These conclude that in any bounded neighborhood of $x$ the series $C(f, h)(x)$ is uniformly convergent.

Since each function sinc $\left[\frac{x-h \underline{k}}{h}\right]$ is right-monogenic, the partial sums $C(f, h)(x)$ are right-monogenic. Owing to Lemma 3.2 and the argument made before the statement of Lemma 3.1, we complete the proof of Theorem 3.4. 
Remark 3.7. For $n>2$ we have a quicker proof of Theorem 3.4 by using the weaker estimate

$$
|\operatorname{sinc}(x)| \leq \frac{C\left(\left|x_{0}\right|\right) e^{\sqrt{n} \pi\left|x_{0}\right|}}{\prod_{j=1}^{n}\left(1+\left|x_{j}\right|\right)^{\frac{n-1}{n}}} \quad \forall x \in \mathbb{R}_{1}^{n} .
$$

Indeed, in the case, if replacing the sum on the right-hand side of inequality (28) by

$$
\sum_{|\underline{k}|>M} \frac{1}{\prod_{j=1}^{n}\left[1+\left|\frac{\left(x_{j}-h k_{j}\right)}{h}\right|\right]^{2 \frac{n-1}{n}}}
$$

the whole proof of Lemma 3.2 is still valid. The assertion of (29) involves to show

$$
\left|I_{1}^{ \pm}(x)\right| \leq \frac{C\left(\left|x_{0}\right|\right) e^{\sqrt{n} \pi\left|x_{0}\right|}}{\left(1+\left|x_{i_{1}}\right|\right) \cdots\left(1+\left|x_{i_{n-1}}\right|\right)} \quad \forall x \in \mathbb{R}_{1}^{n},
$$

with any $1 \leq i_{1}<\ldots<i_{n-1} \leq n$. This can easily be done by using the same integration by parts method used for deducing (15). The method, however, cannot be used to deduce (16), for in the integration by parts the factor $\underline{\xi} /|\xi|$, after being taken partial derivatives $n$ times, will have the magnitude $1 /|\bar{\xi}|^{n}$ that is not locally integrable around $\underline{\xi}=0$ There are $n$ inequalities like $(30)$. Multiplying them together, we obtain

$$
\left|I_{1}^{ \pm}(x)\right| \leq \frac{C\left(\left|x_{0}\right|\right) e^{\sqrt{n} \pi\left|x_{0}\right|}}{\left(1+\left|x_{1}\right|\right)^{\frac{n-1}{n}} \cdots\left(1+\left|x_{n}\right|\right)^{\frac{n-1}{n}}} \quad \forall x \in \mathbb{R}_{1}^{n}
$$

Combining this with (15), we obtain (29).

Remark 3.8. The inequality in Lemma 3.1 has a homogeneous form

$$
|\operatorname{sinc}(x)| \leq \frac{C\left(\left|x_{0}\right|\right) e^{\sqrt{n} \pi\left|x_{0}\right|}}{\prod_{j=1}^{n}\left(\left|x_{0}\right|+\left|x_{j}\right|\right)} \quad \forall x \in \mathbb{R}_{1}^{n},
$$

where $C\left(\left|x_{0}\right|\right)$ is a polynomial of $\left|x_{0}\right|$ with positive coefficients. For $x_{0} \neq 0$, the relations

$$
\frac{\min \left\{1,\left|x_{0}\right|\right\}}{\left|x_{0}\right|+\left|x_{j}\right|} \leq \frac{1}{1+\left|x_{j}\right|} \leq \frac{1+\left|x_{0}\right|}{\left|x_{0}\right|+\left|x_{j}\right|}, \quad j=1, \ldots, n,
$$

show that the inequality (31) is equivalent to (14). For $x_{0}=0$ inequality (14) implies (31), but not vice versa. Hence the homogeneous form is actually weaker. 
The following corollary can be deduced from Theorem 3.4 and the technique in $[4]$.

Corollary 3.9. There hold:

(a) Let

$$
F(x)=\frac{1}{\omega_{n}} \int_{\mathbb{R}^{n}} \operatorname{sinc}(\underline{y}) E(\underline{y}-x) n(\underline{y}) d \underline{y} \quad x \in \mathbb{R}_{+}^{n+1},
$$

where $E(x)=\bar{x} /|x|^{n+1}$ is the Cauchy kernel, and $\omega_{n}=2 \pi^{\frac{n+1}{2}} / \Gamma\left(\frac{n+1}{2}\right)$ is the area of the $n$-dimensional unit sphere in $\mathbb{R}_{1}^{n}$. Then

$$
F(x)=\operatorname{sinc}^{+}(x)=\frac{1}{2}\left(\operatorname{sinc} * P_{x_{0}}\right)(\underline{x})+\frac{1}{2}\left(\operatorname{sinc} * Q_{x_{0}}\right)(\underline{x}),
$$

where

$$
P_{x_{0}}(\underline{x})=\frac{2}{\omega_{n}} \frac{x_{0}}{\left(|\underline{x}|^{2}+x_{0}^{2}\right)^{\frac{n+1}{2}}} \quad \text { and } \quad Q_{x_{0}}(\underline{x})=\frac{2}{\omega_{n}} \frac{\underline{x}}{\left(|\underline{x}|^{2}+x_{0}^{2}\right)^{\frac{n+1}{2}}}
$$

are the Poisson and the conjugate Poisson kernel. In particular, as $x_{0} \rightarrow 0^{+}$, $\left(\operatorname{sinc} * P_{x_{0}}\right)(\underline{x}) \rightarrow \operatorname{sinc}(\underline{x})$ and $\left(\operatorname{sinc} * Q_{x_{0}}\right)(\underline{x}) \rightarrow \sum_{j=1}^{n} R_{j}(\operatorname{sinc})(\underline{x}) e_{j}$, where

$$
R_{j}(f)(\underline{x})=\lim _{\epsilon \rightarrow 0^{+}} \frac{1}{\omega_{n}} \int_{|\underline{y}-\underline{x}|>\epsilon} \frac{x_{j}-y_{j}}{|\underline{x}-\underline{y}|^{n+1}} f(\underline{y}) d \underline{y}
$$

is the jth-Riesz transform of $f$.

(b) Let $\Delta=\sum_{j=0}^{n} \frac{\partial^{2}}{\partial x_{j}^{2}}, \Delta u=0$ in $\mathbb{R}_{+}^{n+1}$, and $u\left(0^{+}+\underline{x}\right)=f(\underline{x})$, where $f$ is the scalar part of a function in $P W\left(\frac{\pi}{h}\right)$. Then, for $x=x_{0}+\underline{x} \in R_{+}^{n+1}$,

$$
u\left(x_{0}+\underline{x}\right)=\frac{1}{2} \sum_{\underline{k} \in \mathbb{Z}^{n}} f(h \underline{k})\left(\operatorname{sinc}\left(\frac{\cdot-h \underline{k}}{h}\right) * P_{x_{0}}\right)(\underline{x}) .
$$

(c) Let $\Delta=\sum_{j=0}^{n} \frac{\partial^{2}}{\partial x_{j}^{2}}, \Delta u=0$ in $\mathbb{R}_{+}^{n+1}$, and $u\left(0^{+}+\underline{x}\right)=\sum_{j=1}^{n} R_{j}(f)(\underline{x}) e_{j}$, where $f$ is the scalar part of a function in $P W\left(\frac{\pi}{h}\right)$ and $R_{j}(f)$ is as in $(32)$. Then, for $x=x_{0}+\underline{x} \in \mathbb{R}_{+}^{n+1}$,

$$
u\left(x_{0}+\underline{x}\right)=\frac{1}{2} \sum_{\underline{k} \in \mathbb{Z}^{n}} f(h \underline{k})\left(\operatorname{sinc}\left(\frac{\cdot-h \underline{k}}{h}\right) * Q_{x_{0}}\right)(\underline{x}) .
$$




\section{References}

[1] Brackx, F., Delanghe, R. and F. Sommen: Clifford Analysis. Research Notes in Mathematics 76. Boston (MA): Pitman 1982.

[2] Gel'fand, I. M. and G. E. Shilov: Generalized Functions. New York: Academic Press 1964.

[3] Kou, K. and T. Qian: Shannon sampling and estimation of band-limited functions in the several complex variables setting (to appear in Acta Mathematica Sinica).

[4] Kou, K. and T. Qian: The Paley-Wiener theorem in $\mathbb{R}^{n}$ with the Clifford analysis setting. J. Func. Anal. 189 (2002), 227 - 241.

[5] Lund, J. and K. Bowers: Sinc Methods for Quadrature and Differential Equations. Philadelphia (PA): SIAM 1992.

[6] Li, C., McIntosh, A. and T. Qian: Clifford algebras, Fourier transforms, and singular convolution operators on Lipschitz surfaces. Rev. Mat. Iberoamericana $10(1994)(3), 665-721$.

[7] Mitrea, M.: Clifford Wavelets, Singular Integrals, and Hardy Spaces. Lecture Notes in Mathematics 1575. Berlin: Springer 1994.

[8] Sommen, F.: Some connections between Clifford analysis and complex analysis. Complex Variables: Theory Appl. (1982)(1), 97 - 118.

[9] Sommen, F.: Plane waves, biregular functions and hypercomplex Fourier analysis. Suppl. Rend. Circ. Mat. Palermo Serie II (1985)(9), pp. $205-219$.

[10] Sommen, F.: Microfunctions with values in a Clifford algebra II. Sci. Papers College Arts Sci. Univ. Tokyo 36 (1986)(1), 15 - 37.

[11] Stenger, F.: Numerical Methods Based on Sinc and Analytic Functions. New York: Springer 1993.

[12] Stein, E. and G. Weiss: Introduction to Fourier Analysis on Euclidean Spaces. Princeton (N.J.): Princeton University Press 1971.

[13] Whittaker, E.: On the functions which are represented by the expansions of the interpolation theory. Proc. Roy. Soc. 35 (1915), $181-194$.

Received 07.07.2004 